\title{
Topological mosaics in moiré superlattices of van der Waals heterobilayers
}

\author{
Qingjun Tong ${ }^{1}$, Hongyi Yu', Qizhong Zhu', Yong Wang ${ }^{1,2}$, Xiaodong $\mathrm{Xu}^{3,4}$ and Wang Yao ${ }^{1 \star}$
}

Van der Waals (vdW) heterostructures formed by twodimensional atomic crystals provide a powerful approach towards designer condensed matter systems ${ }^{1-15}$. Incommensurate heterobilayers with small twisting and/or lattice mismatch lead to the interesting concept of moiré superlattices ${ }^{2-7}$, where the atomic registry is locally indistinguishable from commensurate bilayers but has local-to-local variation over long range. Here we show that such moiré superlattices can lead to periodic modulation of local topological order in vdW heterobilayers formed by two massive Dirac materials. By tuning the $\mathrm{vdW}$ heterojunction from normal to the inverted type-II regime via an interlayer bias, the commensurate heterobilayer can become a topological insulator (TI), depending on the interlayer hybridization controlled by the atomic registry between the vdW layers. This results in a mosaic pattern of $\mathrm{TI}$ regions and normal insulator (NI) regions in moiré superlattices, where topologically protected helical modes exist at the TI/NI phase boundaries. By using symmetry-based $k \cdot p$ and tight-binding models, we predict that this topological phenomenon can be present in inverted transition metal dichalcogenides heterobilayers. Our work points to a new means of realizing programmable and electrically switchable topological superstructures from two-dimensional arrays of $\mathrm{TI}$ nano-dots to one-dimensional arrays of TI nano-stripes.

Two-dimensional (2D) topological insulators (TI), or quantum spin Hall (QSH) insulators ${ }^{16-18}$, feature topologically protected helical edge states with exotic conducting behaviours in the bulk insulating gap. Experimental evidence of such edge states has been extensively explored in the QSH phases of $\mathrm{HgTe} / \mathrm{CdTe}$ and $\mathrm{InAs} / \mathrm{GaSb}$ quantum wells arising from topological band inversion at the heterojunctions ${ }^{19-26}$. Implementation of helical conducting channels may have profound consequences in quantum electronics and spintronics, but such channels exist only at the crystal edge or interface with other normal insulator (NI) materials. The typical planar layout of integrated circuits implies the need to engineer programmable lateral superstructures comprised of distributed TI edges or TI/NI interfaces. Moreover, on/off switching of the QSH phases and the helical channels is also highly desirable ${ }^{27}$. Such systems may open new opportunities for manipulating topological phenomena and enable new functionalities for integrated electronics and spintronics.

Compared to existing crystalline QSH systems ${ }^{18-28}$, vdW heterobilayers formed by stacking $2 \mathrm{D}$ atomic crystals offer several unique possibilities. Some 2D crystals, such as monolayer transition metal dichalcogenides (TMD), endow electrons with Dirac physics ${ }^{29,30}$. Using these $2 \mathrm{D}$ crystals as building blocks indicates an interesting scenario in which to investigate the band inversion of Dirac fermions $s^{31,32}$, for example, an inverted type-II heterojunction as first explored in InAs/GaSb quantum wells ${ }^{22,23}$, but now between massive Dirac cones (that is, in which the upper cone from one layer energetically overlaps and hybridizes with the lower cone from the other layer, see Fig. 1). Tunable heterojunction band alignment by an interlayer bias ${ }^{9-15}$ can then realize the desired on/off switching of the possible topological band inversion. The most interesting possibility comes from engineering superlattices in incommensurate bilayers of a long-period moiré pattern ${ }^{2-7}$. Within a length scale much larger than the lattice constant but small compared to the moiré period, the atomic registry between the vdW layers has negligible difference from commensurate bilayers, while the moiré pattern is a long-range variation in the local registry. With such an atomic registry determining the form of interlayer hybridization, the moiré pattern can realize a superlattice modulation on the local electronic structures, and possibly topological structures.

Here we show that engineering moiré superlattices in heterobilayers of massive Dirac materials can lead to the concept of a programmable topological mosaic (that is, a lateral superstructure modulation of local topological phases). The findings are presented in several steps. First we show that, upon interlayer hybridization of massive Dirac cones in the inverted type-II regime, a commensurately stacked bilayer can become a TI, depending on the form of interlayer hopping, which is controlled by the atomic registry between the layers. This general finding is embodied by the example of inverted TMD heterobilayers, where symmetry dictates multiple TI phases separated by NI phases when the interlayer atomic registry is varied. We then consider incommensurate TMD heterobilayers, in which the formation of the long-period moiré pattern determines the variation in the local atomic registry, and thus corresponds to a pattern of spatial separation of the TI and NI phases. Strain can tune such a topological mosaic from $2 \mathrm{D}$ arrays of TI nano-dots to $1 \mathrm{D}$ arrays of TI nano-stripes. Lastly, we use the tight-binding model to numerically demonstrate the topological mosaic and the topologically protected helical modes at the TI/NI phase boundaries in $1 \mathrm{D}$ moiré superlattices formed by a strained TMD monolayer on an unstrained monolayer.

We start by considering commensurate bilayers of typical Dirac materials with the $2 \mathrm{D}$ hexagonal lattice, where the Dirac cones sit at the $\mathrm{K}$ and $-\mathrm{K}$ corners of the hexagonal Brillouin zone, denoted by the valley index $\tau= \pm 1$. The Dirac cones from the two layers and their coupling can be described by the minimal $\mathbf{k} \cdot \mathbf{p}$ model:

$$
\begin{aligned}
& H_{\tau=1}(\mathbf{q}) \\
& =\left[\begin{array}{cccc}
-\Delta / 2+M_{\mathrm{u}} & v_{\mathrm{u}}\left(q_{x}-i \epsilon q_{y}\right) & t_{\mathrm{cc}} & t_{\mathrm{cv}} \\
v_{\mathrm{u}}\left(q_{x}+i \epsilon q_{y}\right) & -\Delta / 2 & t_{\mathrm{vc}} & t_{\mathrm{vv}} \\
t_{\mathrm{cc}}^{*} & t_{\mathrm{vc}}^{*} & \Delta / 2 & v_{1}\left(q_{x}-i q_{y}\right) \\
t_{\mathrm{cv}}^{*} & t_{\mathrm{vv}}^{*} & v_{1}\left(q_{x}+i q_{y}\right) & \Delta / 2-M_{1}
\end{array}\right]
\end{aligned}
$$

\footnotetext{
${ }^{1}$ Department of Physics and Center of Theoretical and Computational Physics, University of Hong Kong, Hong Kong, China. ${ }^{2}$ School of Physics, Nankai University, Tianjin 300071, China. ${ }^{3}$ Department of Physics, University of Washington, Seattle, Washington 98195 , USA. ${ }^{4}$ Department of Materials Science and Engineering, University of Washington, Seattle, Washington 98195, USA. *e-mail: wangyao@hku.hk
} 
a
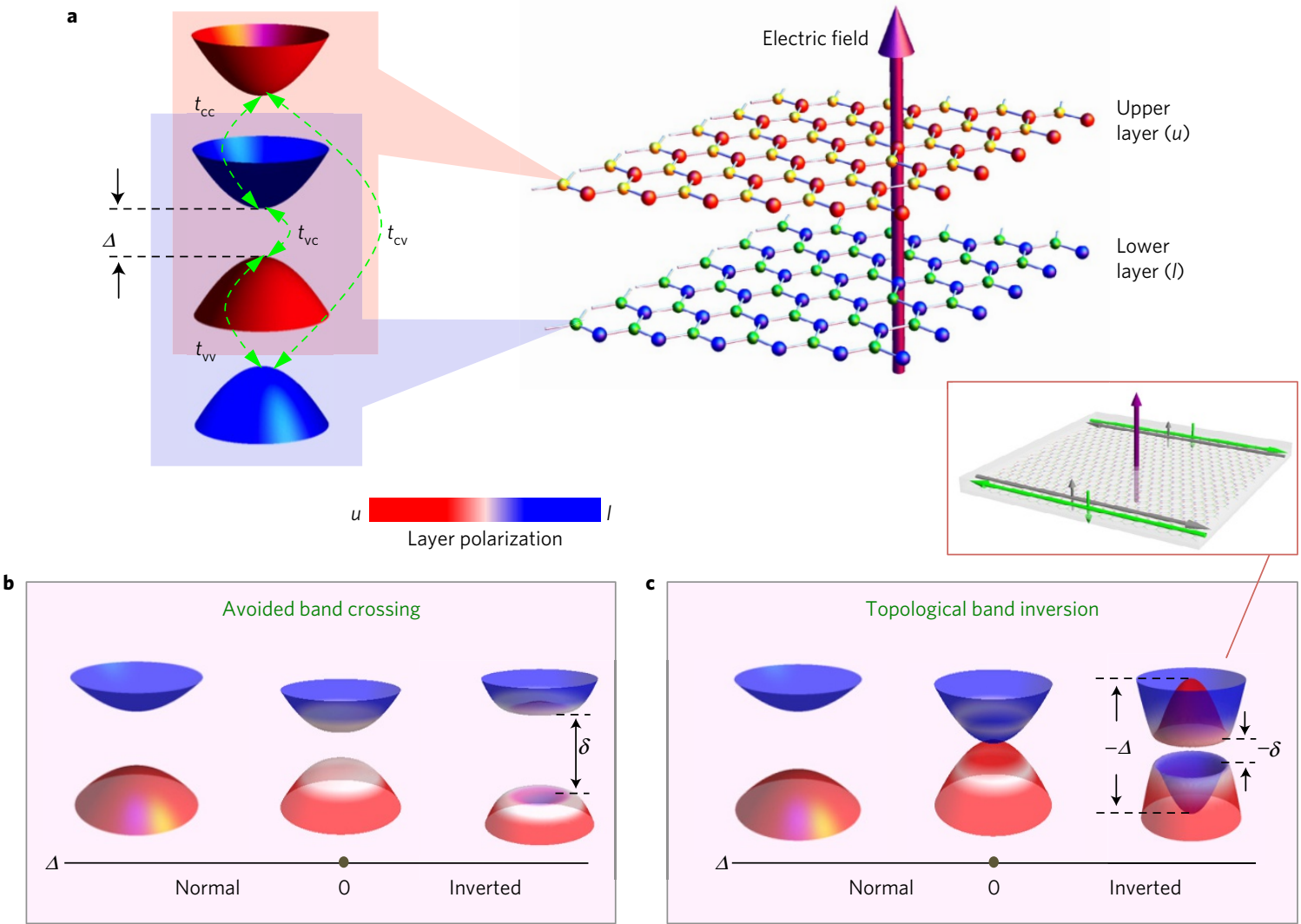

Figure 1 | Electrically controlled band inversion in heterobilayer of massive Dirac materials. a, In type-II band alignment, the heterobilayer band gap $\Delta$, formed between the upper Dirac cone (blue) in layer I and the lower cone (red) in layer $u$, is tunable by a perpendicular electric field. b,c, When the band alignment is tuned from the normal $(\Delta>0)$ to the inverted regime $(\Delta<0)$, depending on the relative strength among the interlayer hopping channels (curved arrows in $\mathbf{a}$ ), the Dirac cones from the two layers hybridize either through avoided band crossing (b) or topological band inversion (c). In the latter case, the electric field can switch on the topological insulating phase where helical edge modes (see inset) then appear in the hybridization gap $\delta$.

Here $M_{\mathrm{u}}\left(M_{1}\right)$ corresponds to the mass and $v_{\mathrm{u}}\left(v_{1}\right)$ is Fermi velocity in the upper (lower) layer. $\epsilon= \pm$ denotes the two types of valley alignment of the Dirac cones in the bilayer, determined by the orientation of stacking. In the example of TMD heterobilayers, $\epsilon=-$ corresponds to H-type stacking (Fig. 2a), while $\epsilon=+$ is for R-type stacking (Fig. 2b). $t_{i j}(i, j=\mathrm{c}, \mathrm{v})$ are the interlayer hopping matrix elements between the $q=0$ (K point) Bloch state in band $i$ of layer $u$ and the one in band $j$ of layer $l . \Delta=\Delta_{\mathrm{g}}-U$, where $U$ is the interlayer bias proportional to the perpendicular electric field, and $\Delta_{\mathrm{g}}$ is the bilayer band gap in the absence of the field (see Supplementary Table 5).

Figure 1a schematically illustrates the type-II heterojunction between the Dirac cones from the two layers. For $\Delta<0$, the bilayer is in the inverted type-II regime-that is, the lower cone in layer $u$ and upper cone in layer $l$ overlap in energy. The cones from the two layers can then hybridize through the four possible interlayer hopping channels, as given in equation (1). We focus on the neighbourhood of the critical point $\Delta=0$. In such a case, $M_{\mathrm{u}}, M_{\mathrm{l}} \gg \Delta$ and the $4 \times 4$ Hamiltonian in equation (1) can be projected to a $2 \times 2$ Hamiltonian,

$$
\begin{aligned}
H(\mathbf{q}) & \cong\left(\frac{\Delta}{2}+\frac{B}{2} q^{2}\right) \hat{\sigma}_{z}+\frac{D}{2} q^{2} \\
& +\left[\hat{\sigma}_{+}\left(t_{\mathrm{vc}}^{*}+\frac{v_{1}}{M_{1}} t_{\mathrm{vv}}^{*} q_{-}-\frac{v_{\mathrm{u}}}{M_{\mathrm{u}}} t_{\mathrm{cc}}^{*} q_{-\epsilon}+\frac{v_{1} v_{\mathrm{u}}}{M_{\mathrm{l}} M_{\mathrm{u}}} t_{\mathrm{cv}}^{*} q_{-\epsilon} q_{-}\right)+\text {h.c. }\right]
\end{aligned}
$$

where $B=\left(\left(v_{\mathrm{u}}^{2} / M_{\mathrm{u}}\right)+\left(v_{1}^{2} / M_{1}\right)\right), D=\left(v_{\mathrm{u}}^{2} / M_{\mathrm{u}}\right)-\left(v_{1}^{2} / M_{1}\right), q \equiv|\mathbf{q}|$, $q_{ \pm} \equiv q_{x} \pm i q_{y}$ and $\hat{\sigma}_{ \pm} \equiv \hat{\sigma}_{x} \pm i \hat{\sigma}_{y}$. The Pauli matrices $\hat{\sigma}$ are spanned by the $q=0$ conduction state in layer $l$ and the valence state in layer $u$.
For the interlayer hopping effect, we retain only up to the leading order term by each hopping channel.

As $\Delta$ is varied from positive to negative by the interlayer bias, the Hamiltonian in equation (2) can have a gap-closing topological phase transition depending on the dominance of the interlayer hopping terms. The $t_{\mathrm{vc}}$ term has a $q$-independent coupling form, so its dominance in the interlayer hybridization leads to the avoided band crossing (gap never closes) where the Hall conductance in the gap remains zero (Fig. 1b). For the R-type stacking $(\epsilon=+)$, the $t_{c c}$ and $t_{\mathrm{vv}}$ terms in equation (2) both have a $q$-linear coupling with a chirality index of 1 , so their dominance leads to a topological band inversion where the Hall conductance changes by a quantized value $-e^{2} / h$ at the gap-closing point (Fig. 1c). The $t_{\mathrm{cv}}$ term has a $q$-quadratic coupling with a chirality index of 2 , so its dominance leads to topological band inversion where the Hall conductance changes by $-2 e^{2} / h$. For the H-type stacking $(\epsilon=-)$, both the $t_{\mathrm{vc}}$ and $t_{\mathrm{cv}}$ terms lead to the trivial avoided crossing, while the $t_{\mathrm{cc}}\left(t_{\mathrm{vv}}\right)$ term leads to a topological band inversion where the Hall conductance changes by $e^{2} / h\left(-e^{2} / h\right)$.

The relative strengths among the different interlayer hopping matrix elements $t_{i j}$ are determined by the atomic configuration, including the orbital composition of the Dirac cones and the lateral registry between the vdW layers. Varying the latter by an interlayer translation therefore becomes a means of control to tune between the different topological phases in the inverted regime. This is demonstrated below using the realistic example of TMD heterobilayers. The valence band edge of the TMD monolayer has a spin up (down) state only at the $K(-K)$ valley because of the giant spin splitting. So only the spin up (down) massive Dirac cones are relevant at the $\mathrm{K}(-\mathrm{K})$ valley. First-principles calculations and 
a

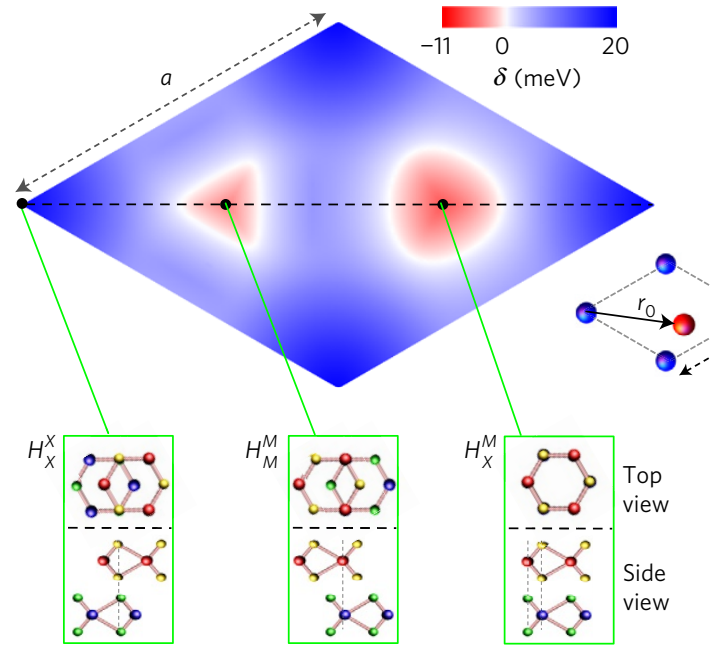

b

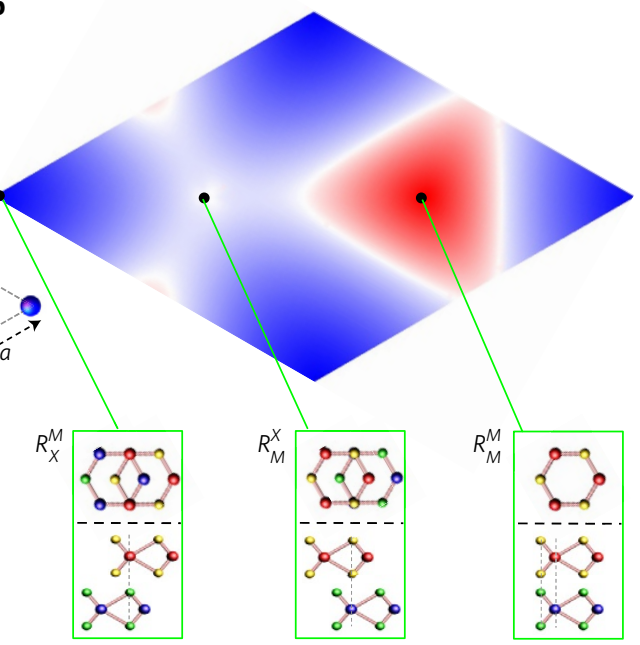

c

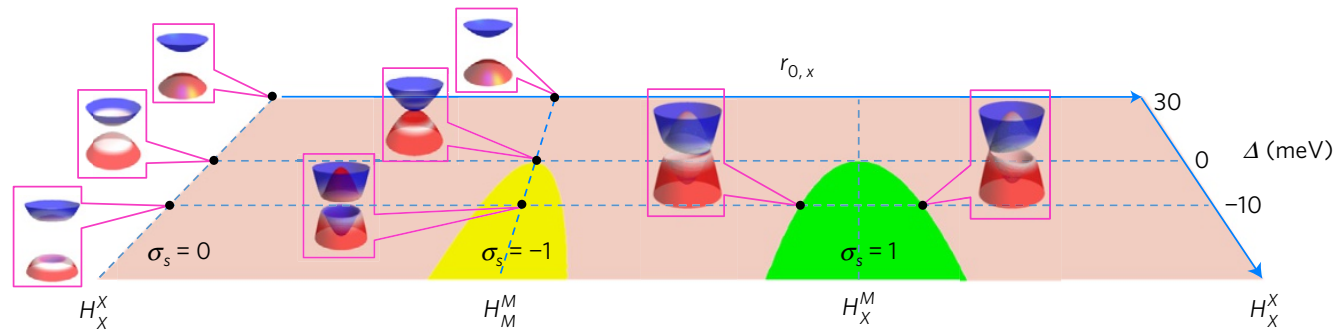

Figure 2 | Topological phase diagrams for commensurate TMD bilayers. a, Hybridization gap $\delta$ as a function of interlayer translation $\boldsymbol{r}_{0}$ in an $\mathrm{H}$-type bilayer (see insets) of inverted type-II alignment ( $\Delta=-40 \mathrm{meV}$ ). The bilayer is a topological (normal) insulator in the red (blue) region of the $\boldsymbol{r}_{0}$ space. Calculation is based on the $\mathbf{k} \cdot \mathbf{p}$ model (equations (1) and (3) in text). $\mathbf{b}$, R-type stacking, also at $\Delta=-40 \mathrm{meV}$. c, Phase diagram parameterized by both $\Delta$ and $r_{0}$ for $\mathrm{H}$-type stacking, where $\boldsymbol{r}_{0}$ is restricted on the dashed line in $\mathbf{a}$. Yellow and green regions are two topological insulating phases, with distinct QSH conductance $\sigma_{s}$ in the hybridization gap. The hybridized Dirac cones at valley $-\mathrm{K}$ at a few representative phase space points are shown in the insets.

experiments have shown that certain TMD heterobilayers can be tuned into the inverted regime by a moderately strong interlayer bias $^{33,34}$ (see Supplementary Table 5).

We first analyse high-symmetry stacking configurations of the $\mathrm{H}$-type (that is, two layers have opposite orientations). If either the metal $(M)$ or chalcogen $(X)$ sites of layer $u$ are vertically aligned with the $M$ or $X$ sites of layer $l$, then $\mathrm{C}_{3}$ rotational symmetry is retained. For the three high-symmetry H-configurations (Fig. 2a), the rotational symmetry of the Bloch states at $\pm K$ dictates that certain interlayer hopping channels must vanish, as listed in Table 1. It is then clear from equation (2) that inverted TMD heterobilayers of $H_{M}^{M}$ stacking form a topological insulator with a quantized spin Hall conductance $\sigma_{s}=-1$ (in units of $e^{2} / h$ ) in the hybridization gap. TMD heterobilayers of $H_{X}^{M}$ stacking also form a topological insulator, but with an opposite spin Hall conductance $\left(\sigma_{s}=1\right)$, while $H_{X}^{X}$ heterobilayers form a normal insulator $\left(\sigma_{s}=0\right)$.

All stacking configurations of the same orientation can span a phase space parameterized by the interlayer translation $\mathbf{r}_{0}$, defined as the lateral displacement between two metal sites from the two layers, respectively, which takes values within a unit cell. With the configurations $H_{M}^{M}, H_{X}^{M}$ and $H_{X}^{X}$ being three separated points in this phase space (Fig. 2a), the conclusion that they have distinct QSH conductance from the above symmetry analysis means the inverted TMD bilayer undergoes topological phase transitions as $\mathbf{r}_{0}$ is varied.

With these symmetry-dictated qualitative features of the topological phase diagram, the remaining details are determined by the dependence of interlayer hopping $t_{i j}$ on the interlayer translation $\mathbf{r}_{0}$. In TMD bilayers, the conduction states near $q=0$ are predominantly from the $\mathrm{M} d$-orbital with magnetic quantum number $m=0$, while
Table 1 | Interlayer hopping channels and QSH conductance in the inverted band of commensurate TMD bilayers with 3-fold rotational symmetry.

\begin{tabular}{lllllr} 
& $\boldsymbol{t}_{\mathrm{cc}}$ & $\boldsymbol{t}_{\mathrm{vv}}$ & $\boldsymbol{t}_{\mathrm{cv}}$ & $\boldsymbol{t}_{\mathrm{vc}}$ & $\boldsymbol{\sigma}_{\boldsymbol{s}}$ \\
\hline$R_{M}^{M}$ & $3 T_{0}^{0}$ & $3 T_{2}^{2}$ & 0 & 0 & -1 \\
$R_{X}^{M}$ & 0 & 0 & 0 & $3 T_{0}^{2}$ & 0 \\
$R_{M}^{X}$ & 0 & 0 & $3 T_{2}^{0}$ & 0 & $*$ \\
$H_{X}^{M}$ & 0 & $3 T_{2}^{-2}$ & 0 & 0 & 1 \\
$H_{M}^{M}$ & $3 T_{0}^{0}$ & 0 & 0 & 0 & -1 \\
$H_{X}^{X}$ & 0 & 0 & $3 T_{2}^{0}$ & $3 T_{0}^{-2}$ & 0 \\
\hline
\end{tabular}

$R_{v}^{\mu}\left(H_{v}^{\mu}\right)$ denotes an R-type (H-type) stacking with $\mu$-sites of layer $u$ vertically aligned with $v$-sites of layer $I$, where $\mu, v=M$ or $X$. See Fig. $2 . t_{i j}(i, j=c, v)$ are the interlayer hopping matrix elements between the Bloch state at the $\mathrm{K}$ point in band $i$ of layer $u$ and the one in band $j$ of layer $I . \sigma_{s}$ is the spin Hall conductance, in units of $e^{2} / h$, in the hybridization gap of the inverted heterobilayer. *We note that the massive Dirac model in equation (1) leads to $\sigma_{s}=-2$ through a high-order effect, but the value of $\sigma_{\mathrm{s}}$ can be corrected in the presence of other bands (see

Supplementary Information)

the valence states at valley $\tau$ are from the M $d$-orbital with $m=2 \tau$. The $\mathbf{r}_{0}$ dependence of $t_{i j}$ can be approximated as ${ }^{8}$

$$
\begin{array}{r}
t_{\mathrm{cc}}\left(\mathbf{r}_{0}\right) \cong T_{0}^{0}\left(e^{i \mathbf{K} \cdot \mathbf{r}_{0}}+e^{i \hat{C}_{3} \mathbf{K} \cdot \mathbf{r}_{0}}+e^{i \hat{C}_{3}^{2} \mathbf{K} \cdot \mathbf{r}_{0}}\right) \\
t_{\mathrm{vc}}\left(\mathbf{r}_{0}\right) \cong T_{0}^{2 \epsilon}\left(e^{i \mathbf{K} \cdot \mathbf{r}_{0}}+e^{i\left(\hat{C}_{3} \mathbf{K} \cdot \mathbf{r}_{0}+2 \epsilon \pi / 3\right)}+e^{i\left(\hat{C}_{3}^{2} \mathbf{K} \cdot \mathbf{r}_{0}-2 \epsilon \pi / 3\right)}\right) \\
t_{\mathrm{cv}}\left(\mathbf{r}_{0}\right) \cong T_{2}^{0}\left(e^{i \mathbf{K} \cdot \mathbf{r}_{0}}+e^{i\left(\hat{C}_{3} \mathbf{K} \cdot \mathbf{r}_{0}-2 \pi / 3\right)}+e^{i\left(\hat{C}_{3}^{2} \mathbf{K} \cdot \mathbf{r}_{0}+2 \pi / 3\right)}\right) \\
t_{\mathrm{vv}}\left(\mathbf{r}_{0}\right) \cong T_{2}^{2 \epsilon}\left(e^{i \mathbf{K} \cdot \mathbf{r}_{0}}+e^{i\left(\hat{C}_{3} \mathbf{K} \cdot \mathbf{r}_{0}+(1-\epsilon) \pi / 3\right)}+e^{i\left(\hat{C}_{3}^{2} \mathbf{K} \cdot \mathbf{r}_{0}-(1-\epsilon) \pi / 3\right)}\right)
\end{array}
$$




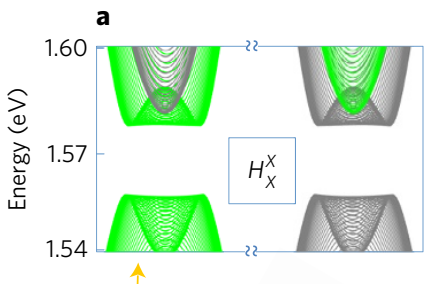

b

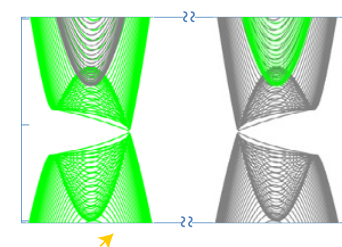

f
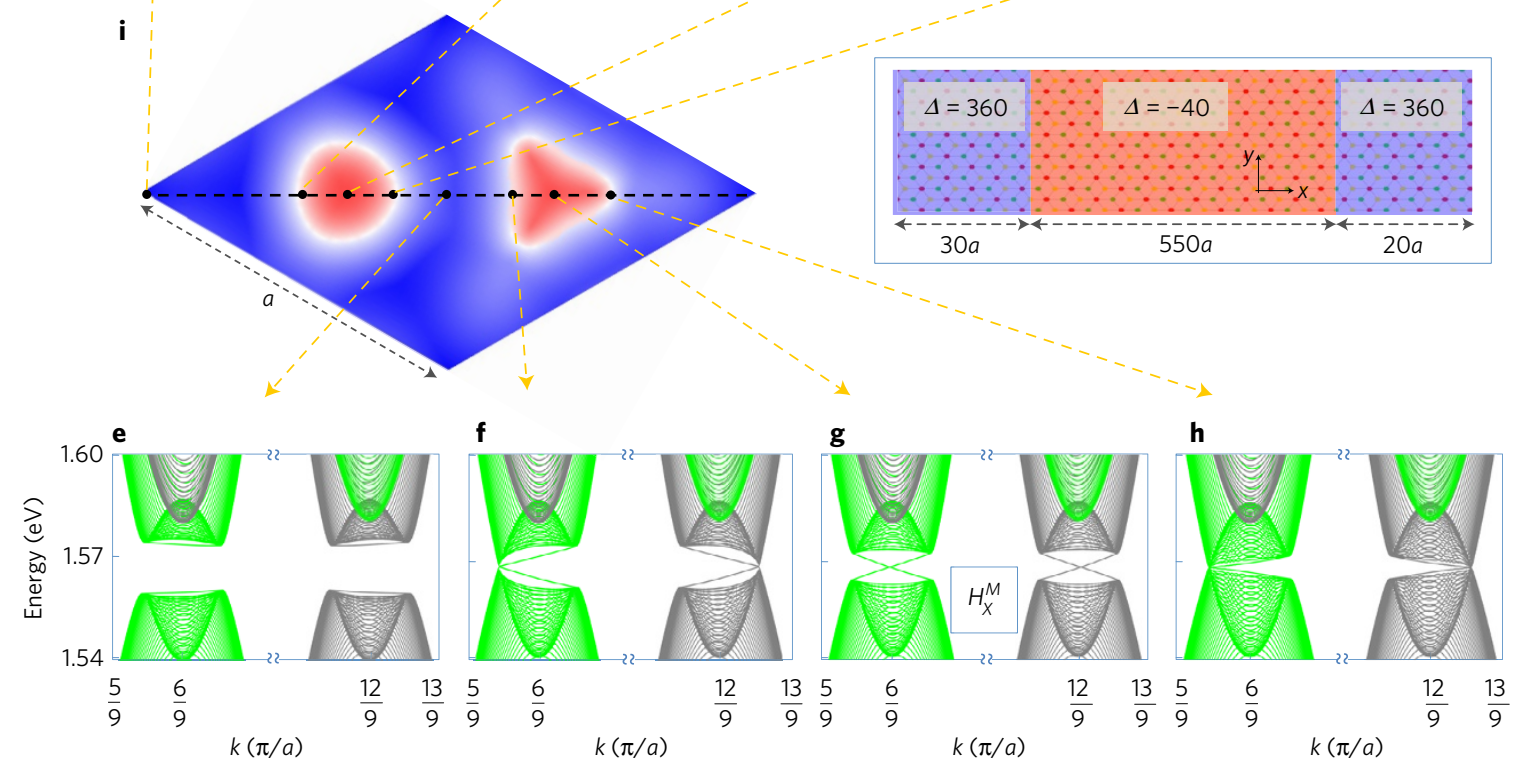

c

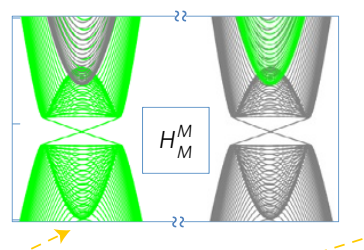

d

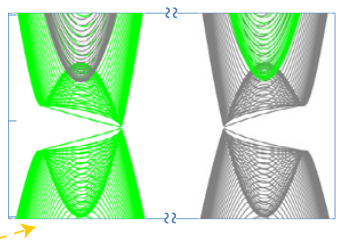

Figure 3 | Bulk-edge correspondence in the topological phase diagram. a-h, Bulk bands and corresponding edge state dispersions in inverted H-type TMD bilayers with various interlayer translation (black dots in i). Grey and green colours denote the spin up and down bands, respectively. i, Bulk hybridization gap $\delta$ from the tight-binding calculation. The sign of $\delta$ is determined by whether there exist gapless edge states. The inset illustrates the set-up of the tight-binding calculation. The inverted region with $\Delta=-40 \mathrm{meV}$ is sandwiched between normal regions with a large gap $\Delta=360 \mathrm{meV}$. The spectra in $\mathbf{a}-\mathbf{h}$ are within the gap of the normal region. The gapless helical modes in the hybridization gap in $\mathbf{c}$ and $\mathbf{g}$ are localized at the inverted/normal interfaces.

where $\mathbf{K}, \hat{C}_{3} \mathbf{K}$ and $\hat{C}_{3}^{2} \mathbf{K}$ are respectively the wavevectors for the three $\mathrm{K}$ corners of the first Brillouin zone. $T_{m}^{m^{\prime}}$ denotes the Fourier component at $\mathbf{K}$ of the interlayer hopping integral between a $d_{m^{\prime}}$ orbital in layer $u$ and a $d_{m}$ orbital in layer $l$. Note that we derived equation (3) under the two-centre approximation and have dropped Umklapp terms, which are generally expected to be weak (see Supplementary Text Ib). As shown later, quantitative details subject to this approximation are nonessential to the conclusions, as long as $t_{i j}\left(\mathbf{r}_{0}\right)$ observes the $\mathrm{C}_{3}$ symmetry-dictated behaviour including those listed in Table 1.

Figure 2a shows the phase diagram for the inverted H-type TMD bilayers. In the calculations, $T_{0}^{0}=6.7 \mathrm{meV}, T_{0}^{-2}=T_{2}^{0}=3.3 \mathrm{meV}$, and $T_{2}^{-2}=10 \mathrm{meV}$, which are estimated from the first-principles band structures of the TMD homobilayers ${ }^{35,36}$ (see Supplementary Text Ic). The colour map plots the hybridization gap $\delta$ (see Fig. 1b,c), which is taken as negative (positive) if the Hall conductance is quantized (zero) in the gap. There are two isolated (red) regions of negative $\delta$ (that is, TI phases) centred respectively at the $H_{M}^{M}$ and $H_{X}^{M}$ points. The remainder (blue) is a region of positive $\delta$ (that is, NI). Figure $2 c$ shows the electrically controlled topological phase transition for the stacking configurations along the dashed horizontal line in Fig. 2a. The TI phases start to appear at $\Delta=0$, at the high-symmetry $H_{M}^{M}$ and $H_{X}^{M}$ points, while the size of the TI phase regions grows with the magnitude of the inverted gap $(|\Delta|)$. The insets are the hybridized Dirac cones calculated from equation (1) at various values of interlayer translation and $\Delta$, which clearly illustrate the avoided band crossing in the $H_{X}^{X}$ TMD bilayer and the topological band inversion in the $H_{M}^{M}$ TMD bilayer as $\Delta$ changes, as well as the asymmetric band touching that takes place upon the topological phase transition in TMD bilayers of lowsymmetry stacking.

For the R-type stacking (two layers have same orientations), there is a similar topological phase diagram. For the high-symmetry $R_{M}^{M}$ stacking configuration, $t_{\mathrm{cc}}$ and $t_{\mathrm{vv}}$ are the only symmetry-allowed interlayer hopping channels in equation (1), which dictate the inverted TMD heterobilayer to be a TI with $\sigma_{s}=-1$. The $R_{X}^{M}$ TMD bilayer is always a NI. Figure $2 \mathrm{~b}$ shows the calculated phase diagram based on equations (1) and (3), where we find a TI phase (red) region and a NI phase (blue) region centred, respectively, at the $R_{M}^{M}$ and $R_{X}^{M}$ points. Equation (1) also predicts that the $R_{M}^{X}$ TMD bilayer is a TI with $\sigma_{s}=-2$ in the inverted regime. However, compared to the other TI phases, the topological band inversion at $R_{M}^{X}$ is through a weak higher-order effect (see equation (2)). When other interlayer hopping channels through bands beyond the massive Dirac cones are considered, the $R_{M}^{X}$ TMD bilayer in the inverted regime can become a TI with a different $\sigma_{s}$ value (see Supplementary Text IIb).

To demonstrate the QSH edge states in the TI phases, we turn to tight-binding (TB) calculations. Our bilayer TB model is generalized from the nearest-neighbour three-orbital TB model for the monolayer ${ }^{37}$, by adding interlayer hopping that observes the rotational symmetry of the $d$-orbitals (see Supplementary Text IIa). For simplicity, the two-centre interlayer integrals used are shortrange ones allowing hopping to three neighbouring metal sites in the opposite layer only. With this simplification, Umklapp terms have a pronounced contribution to the band-edge hopping matrix elements $t_{i j}$, which results in different $\mathbf{r}_{0}$-dependences from equation (3) (see Supplementary Fig. 2). Nevertheless, comparing the phase diagrams from the $\mathbf{k} \cdot \mathbf{p}$ (Fig. 2a) and from these TB 
a
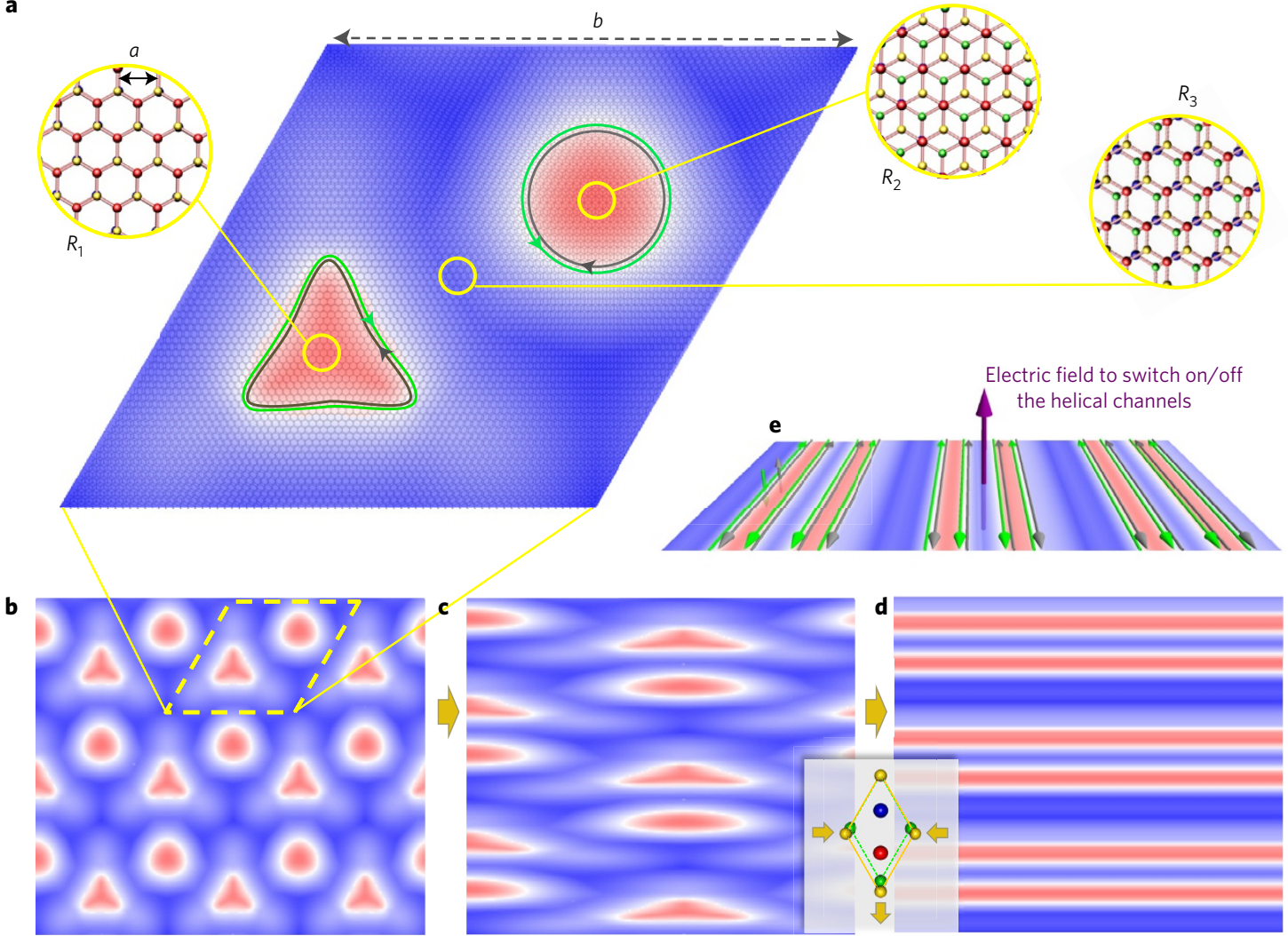

Figure 4 | Topological mosaic in long-period moiré pattern. a, At three locations (yellow circles) in a moiré supercell, in a length scale $\gg$ a (lattice constant) and $\ll b$ (moiré period), local atomic registries are shown which have negligible difference from commensurate TMD bilayers with a corresponding interlayer translation $\boldsymbol{r}_{0}$. Different local regions are thus in either the topological (red) or normal (blue) insulating phase, depending on the local $\boldsymbol{r}_{0}$ value. Grey and green colours denote, respectively, the spin up and down helical modes expected at the TI/NI phase boundaries. $\mathbf{b}-\mathbf{d}$, Evolution of the topological phase separation patterns, when the lattice mismatch in an armchair (zigzag) direction is increased (reduced) by an uniaxial tensile strain on one layer (see inset). e, The electrically switchable dense array of protected helical channels can be exploited for a field effect transistor.

calculations (Fig. 3i), all the important features are in excellent agreement, including the locations and the areas of the TI phase regions and the values of the QSH conductance. This shows that the topological phase diagram is determined by the symmetry in the TMD bilayer, rather than the quantitative details of the models.

Using the TB model, we can examine electronic structures at the edges or interfaces with normal insulating regimes. Figure $3 a-h$ shows the TB calculations for several H-type TMD bilayers in the inverted regime, demonstrating the evolution of the bands and the emergence of the QSH edge states in the hybridization gap as the interlayer translation is varied across the TI/NI phase boundaries.

Now we turn to incommensurate TMD heterobilayers, where lattice mismatch and twisting lead to moiré patterns. The moiré supercell period can reach $\sim 10-100 \mathrm{~nm}$ in TMD heterobilayers of small twisting angles, depending on the choices of the compounds. In such long-period moiré patterns, within a length scale smaller than the moire period $(b)$ but much larger than the lattice constant $(a)$, the local atomic registry has negligible difference from a commensurate TMD bilayer of a certain interlayer translation $\mathbf{r}_{0}$ (see insets in Fig. 4a). Studies of twisted bilayer graphene and graphene/hexagonal boron nitride $(\mathrm{hBN})^{7,38,39}$ have shown that, in the limit of moiré period $\gg$ lattice constant, the local electronic structure at any location $\mathbf{R}$ is described well by bands of commensurate bilayers with the corresponding interlayer translation $\mathbf{r}_{0}(\mathbf{R})$, which, in the present context of TMD bilayers, are topologically nontrivial (trivial) if the local hybridization gap $\delta\left(\mathbf{r}_{0}\right)$ is negative (positive). For example, for the three magnified local regions in Fig. $4 \mathrm{a}, R_{1}$ and $R_{2}$ have the electronic structure of the TI phase, while $R_{3}$ has that of the NI phase. Therefore, topological phase separations are expected in the moiré superlattice. The colour maps in Fig. 4 are plots of $\delta\left(\mathbf{r}_{0}(\mathbf{R})\right)$, the position-dependent hybridization gap from this local approximation, in various moiré patterns which form superlattice modulations of the local topological order.

When the moiré pattern is formed between two undistorted hexagonal lattices, the phase separation in a moiré supercell is isomorphic to the topological phase diagram of the commensurate TMD bilayer parameterized by $\mathbf{r}_{0}$ (Figs 2 and 3). Figure $4 \mathrm{a}, \mathrm{b}$ shows the topological phase separation in such a moiré pattern, realizing periodic arrays of TI nano-dots, where helical modes circulating along the phase boundaries are expected inside the bulk hybridization gap. The variation of the local atomic registry in the moiré pattern, as described by the function $\mathbf{r}_{0}(\mathbf{R})$, can be tuned by the twisting angle as well as the strain. This further allows the tuning of the pattern of topological phase separations, for example, from $2 \mathrm{D}$ arrays of TI nano-dots in Fig. $4 \mathrm{~b}$ to $1 \mathrm{D}$ arrays of TI nano-stripes in Fig. 4d.

We numerically demonstrate the topological mosaic and the topologically protected helical modes at the TI/NI phase boundaries using TB calculations. Here we consider H-type stacking of two TMD layers with identical lattice period in the $y$ (zigzag) direction and a lattice mismatch $\eta$ in the $x$ (armchair) direction. In such 1D moiré superlattices, the local approximation predicts arrays of TI nano-stripes (see Fig. 4d). The two-centre integrals used in the TB calculations here are the same as those used for Fig. 3, but generate locally different interlayer hopping due to the local-tolocal variation of atomic registry. Compared to the calculations in Fig. 3, we have used larger interlayer hopping integrals $T_{0}^{0}=20 \mathrm{meV}, T_{0}^{-2}=T_{2}^{0}=10 \mathrm{meV}$, and $T_{2}^{-2}=30 \mathrm{meV}$ (achievable at 
a
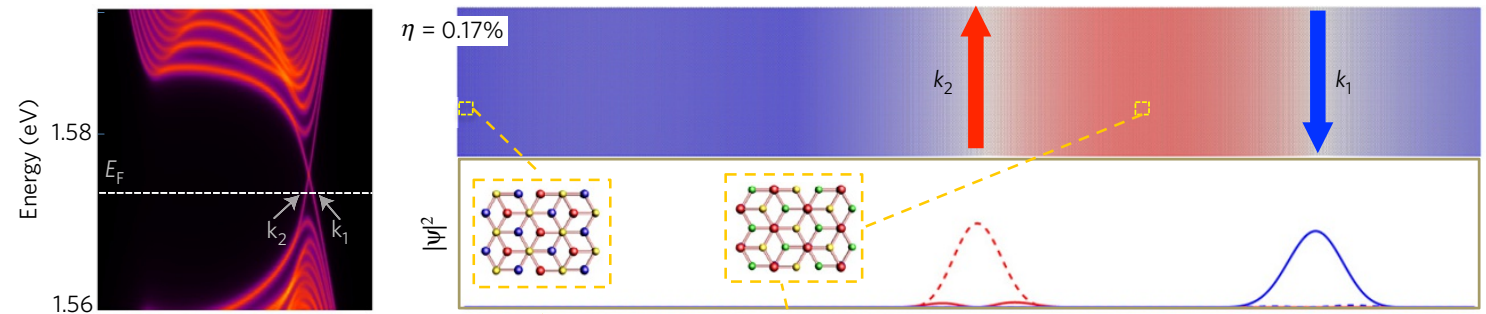

b
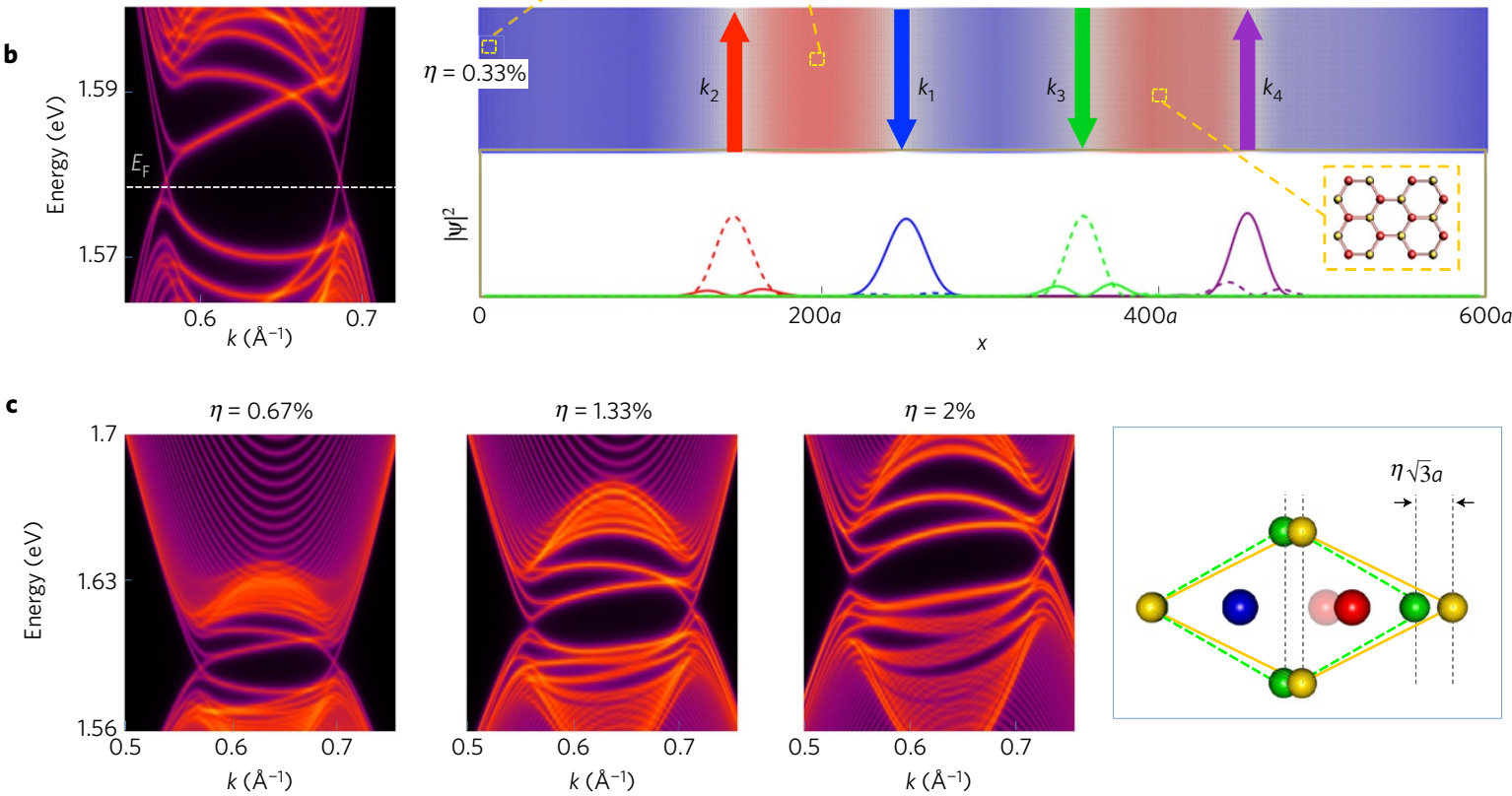

Figure 5 | Topologically protected helical modes at the boundaries of TI nano-stripes in 1D moiré superlattices. The two layers have identical lattice periods in the $y$ (zigzag) direction, while in the $x$ (armchair) direction they have a lattice mismatch $\eta$, as defined in the inset. a, (Left) Energy spectra as a

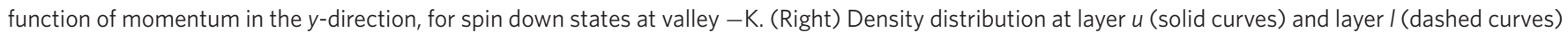
of two in-gap states at energy $E_{\mathrm{F}}$ with momenta $k_{1}$ (blue) and $k_{2}$ (red), respectively, with the colour map above plotting $\delta\left(\boldsymbol{r}_{0}(\boldsymbol{R})\right)$ from the local approximation (see text). $\mathbf{b}$, Same as $\mathbf{a}$, but at a larger value of $\eta$. $E_{\mathrm{F}}$ (horizontal line) crosses the in-gap dispersions from right to left at $k_{1}, k_{2}, k_{3}$ and $k_{4}$. c, With increasing $\eta$, the in-gap modes become gapped at their crossing point in the energy-momentum space, due to the finite size of the TI stripes.

a smaller interlayer distance; see Supplementary Text I), to allow a larger hybridization gap to examine the behaviours of the in-gap modes. The strain effect on intralayer hopping due to change in the atomic positions is also incorporated (see Supplementary Text III).

Figure 5 shows the calculated energy spectra as functions of the wavevector $k$ in the translational invariant $y$-direction, for moiré superlattices with various lattice mismatch $\eta$ in the $x$ direction. For small $\eta$ values, gapless helical modes are indeed found in the gap of the bulk spectra (see Fig. 5a,b). For the helical modes at an ingap energy $E_{F}$, the distribution in the $x$ direction is plotted, together with the spatial colour map of the local hybridization gap $\delta\left(\mathbf{r}_{0}(\mathbf{R})\right)$. These modes, labelled by their wavevectors in the $y$-direction $\left(k_{1}, k_{2}\right.$, $\left.k_{3}, k_{4}\right)$, are all localized at $x$ values corresponding to the zeros of $\delta\left(\mathbf{r}_{0}(\mathbf{R})\right)$. The gapless modes at valley $-\mathrm{K}$ are all spin down states, and their time-reversal counterparts are at valley $\mathrm{K}$. Their chirality is consistent with the QSH conductance associated with $\delta\left(\mathbf{r}_{0}(\mathbf{R})\right.$ ) (see Fig. 2c and Table 1). This confirms the topological phase separations in these long-period moiré, where locations of the TI/NI boundaries and the helical modes are predicted well by $\delta\left(\mathbf{r}_{0}(\mathbf{R})\right)$ from the local approximation.

Figure $5 c$ shows the evolution of the spectra with increasing $\eta$. The change in band alignment arises from the effect of strain on the intralayer hopping. When the moiré period (and hence the width of the TI nano-stripes) is not large enough, the helical modes on different sides of a nano-stripe can couple, leading to a finite gap at their crossing in the energy-momentum space, as shown in Fig. 5c. Through such a finite-size effect, the helical modes at the TI/NI phase boundaries in long-period moiré will eventually evolve into minibands in short-period moiré.

Data availability. The data that support the plots within this paper and other findings of this study are available from the corresponding author upon reasonable request.

Received 14 July 2016; accepted 27 October 2016;

published online 28 November 2016

\section{References}

1. Geim, A. K. \& Grigorieva, I. V. Van der Waals heterostructures. Nature 499, 419-425 (2013)

2. Ponomarenko, L. A. et al. Cloning of Dirac fermions in graphene superlattices. Nature 497, 594-597 (2013).

3. Dean, C. R. et al. Hofstadter's butterfly and the fractal quantum Hall effect in moiré superlattices. Nature 497, 598-602 (2013).

4. Hunt, B. et al. Massive Dirac fermions and Hofstadter butterfly in a van der Waals heterostructure. Science 340, 1427-1430 (2013).

5. Gorbachev, R. V. et al. Detecting topological currents in graphene superlattices. Science 346, 448-451 (2014).

6. Song, J. C. W., Samutpraphoot, P. \& Levitov, L. S. Topological Bloch bands in graphene superlattices. Proc. Natl Acad. Sci. USA 112, 10879-10883 (2015).

7. Jung, J., Raoux, A., Qiao, Z. \& MacDonald, A. H. Ab initio theory of moiré superlattice bands in layered two-dimensional materials. Phys. Rev. B 89, 205414 (2014)

8. Yu, H., Wang, Y., Tong, Q., Xu, X. \& Yao, W. Anomalous light cones and valley optical selection rules of interlayer excitons in twisted heterobilayers. Phys. Rev. Lett. 115, 187002 (2015) 
9. Rivera, P. et al. Valley-polarized exciton dynamics in a $2 \mathrm{D}$ semiconductor heterostructure. Science 351, 688-691 (2016).

10. Fang, H. et al. Strong interlayer coupling in van der Waals heterostructures built from single-layer chalcogenides. Proc. Natl Acad. Sci. USA 111, 6198-6202 (2014).

11. Chiu, M.-H. et al. Spectroscopic signatures for interlayer coupling in $\mathrm{MoS}_{2}-\mathrm{WSe}_{2}$ van der Waals stacking. ACS Nano 8, 9649-9656 (2014).

12. Lee, C.-H. et al. Atomically thin $\mathrm{p}-\mathrm{n}$ junctions with van der Waals heterointerfaces. Nat. Nanotech. 9, 676-681 (2014).

13. Furchi, M. M., Pospischil, A., Libisch, F., Burgdörfer, J. \& Mueller, T. Photovoltaic effect in an electrically tunable van der Waals heterojunction. Nano Lett. 14, 4785-4791 (2014).

14. Cheng, R. et al. Electroluminescence and photocurrent generation from atomically sharp $\mathrm{WSe}_{2} / \mathrm{MoS}_{2}$ heterojunction p-n diodes. Nano Lett. 14, 5590-5597 (2014).

15. Hong, X. et al. Ultrafast charge transfer in atomically thin $\mathrm{MoS}_{2} / \mathrm{WS}$ heterostructures. Nat. Nanotech. 9, 682-686 (2014).

16. Qi, X.-L. \& Zhang, S.-C. Topological insulators and superconductors. Rev. Mod. Phys. 83, 1057-1110 (2011)

17. Hasan, M. Z. \& Kane, C. L. Colloquium: topological insulators. Rev. Mod. Phys. 82, 3045-3067 (2010).

18. Kane, C. L. \& Mele, E. J. Quantum spin Hall effect in graphene. Phys. Rev. Lett. 95, 226801 (2005).

19. Bernevig, B. A., Hughes, T. L. \& Zhang, S.-C. Quantum spin Hall effect and topological phase transition in HgTe quantum wells. Science 314, 1757-1761 (2006).

20. König, M. et al. Quantum spin Hall insulator state in HgTe quantum wells. Science 318, 766-770 (2007).

21. Ma, E. Y. et al. Unexpected edge conduction in mercury telluride quantum wells under broken time-reversal symmetry. Nat. Commun. 6, 7252 (2015).

22. Liu, C., Hughes, T. L., Qi, X.-L., Wang, K. \& Zhang, S.-C. Quantum spin Hall effect in inverted type-II semiconductors. Phys. Rev. Lett. 100, 236601 (2008).

23. Knez, I., Du, R.-R. \& Sullivan, G. Evidence for helical edge modes in inverted InAs/GaSb quantum wells. Phys. Rev. Lett. 107, 136603 (2011).

24. Karalic, M. et al. Experimental evidence for the topological insulator phase in InAs/GaSb coupled quantum wells. Preprint at http://arXiv.org/abs/1606.03627 (2016).

25. Nichele, F. et al. Edge transport in the trivial phase of InAs/GaSb. New J. Phys. 18, 083005 (2016).

26. Suzuki, K., Harada, Y., Onomitsu, K. \& Muraki, K. Edge channel transport in the InAs/GaSb topological insulating phase. Phys. Rev. B 87, 235311 (2013).

27. Qian, X., Liu, J., Fu, L. \& Li, J. Quantum spin Hall effect in two-dimensiona transition metal dichalcogenides. Science 346, 1344-1347 (2014).

28. Abanin, D. A., Lee, P. A. \& Levitov, L. S. Spin-filtered edge states and quantum Hall effect in graphene. Phys. Rev. Lett. 96, 176803 (2006).

29. Xiao, D., Liu, G.-B., Feng, W., Xu, X. \& Yao, W. Coupled spin and valley physics in monolayers of $\mathrm{MoS}_{2}$ and other group-VI dichalcogenides. Phys. Rev. Lett. 108, $196802(2012)$
30. Mak, K. F., McGill, K. L., Park, J. \& McEuen, P. L. The valley Hall effect in $\mathrm{MoS}_{2}$ transistors. Science 344, 1489-1492 (2014).

31. Sie, E. J. et al. Valley-selective optical Stark effect in monolayer $\mathrm{WS}_{2}$. Nat. Mater. 14, 290-294 (2014).

32. Claassen, M., Jia, C., Moritz, B. \& Devereaux, T. P. All-optical materials design of chiral edge modes in transition-metal dichalcogenides. Nat. Commun. 7, 13074 (2016).

33. Gong, C. et al. Band alignment of two-dimensional transition metal dichalcogenides: application in tunnel field effect transistors. Appl. Phys. Lett. 103, 053513 (2013).

34. Chiu, M.-H. et al. Determination of band alignment in the single-layer $\mathrm{MoS}_{2} / \mathrm{WSe}_{2}$ heterojunction. Nat. Commun. 6, 7666 (2015).

35. Liu, K. et al. Evolution of interlayer coupling in twisted molybdenum disulfide bilayers. Nat. Commun. 5, 4966 (2014).

36. Gong, Z. et al. Magnetoelectric effects and valley-controlled spin quantum gates in transition metal dichalcogenide bilayers. Nat. Commun. 4, 2053 (2013).

37. Liu, G.-B., Shan, W.-Y., Yao, Y., Yao, W. \& Xiao, D. Three-band tight-binding model for monolayers of group-VIB transition metal dichalcogenides. Phys. Rev. B 88, 085433 (2013)

38. Lopes dos Santos, J. M. B., Peres, N. M. R. \& Neto, A. H. C. Graphene bilayer with a twist: electronic structure. Phys. Rev. Lett. 99, 256802 (2007)

39. Mele, E. J. Band symmetries and singularities in twisted multilayer graphene. Phys. Rev. B 84, 235439 (2011).

\section{Acknowledgements}

The work is mainly supported by the Croucher Foundation (Croucher Innovation Award), the Research Grants Council and University Grants Committee of Hong Kong (HKU17312916, AoE/P-04/08), and the University of Hong Kong (ORA). Y.W. is partly supported by the NSFC with Grant No. 11604162 and Grant No. 61674083. X.X. is supported by the Department of Energy, Basic Energy Sciences, Materials Sciences and Engineering Division (DE-SC0008145 and SC0012509), and the Cottrell Scholar Award.

\section{Author contributions}

W.Y. conceived and designed the research. Q.T. and H.Y. performed the calculations. Q.T., H.Y. and W.Y. analysed the results with input from Q.Z. and X.X. Y.W. provided support with first-principles calculations. W.Y., Q.T., H.Y. and X.X. prepared the manuscript.

\section{Additional information}

Supplementary information is available in the online version of the paper. Reprints and permissions information is available online at www.nature.com/reprints.

Correspondence and requests for materials should be addressed to W.Y.

\section{Competing financial interests}

The authors declare no competing financial interests. 